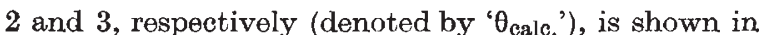
the accompanying graph. This shows that only when $n=2$ does $\theta_{\text {calc }}$ fit closely the experimental

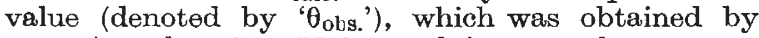
assuming that $5 \times 10^{14}$ metal ions or the average numbers of metal ions per square centimetre of the adsorbent surface are equally capable of chemisorbing each dissociated particle. The result is obviously in contrast to that for nickel quoted above. A similar result was also obtained in the case of copper chromite and zine chromite, as shown in the accompanying table.

As can be seen from the table, if we assume that $n=1$ or 3 , the fraction covered, or $\theta_{\text {calc., fails to }}$ account entirely for the experimental value $\left(\theta_{\mathrm{ots}}\right)$ for any of the oxide catalysts, whereas $\theta_{\text {calc. }}$ corresponding to $n=2$ gives a good fit. Quite apart from the numerical agreement between $\theta_{\text {calc. }}$ and $\theta_{\text {obs. }}$. in the latter case, it seems very likely that the carbon dioxide molecule is chemisorbed with partial dissociation into two particles, that is, presumably into chemisorbed carbon monoxide and oxygen atoms.

Detailed accounts of this work will be published in J. Res. Inst. Catalysis, Hokk. Univ. Our thanks are due to Prof. J. Horiuti for his advice.

Research Institute for Catalysis,

\section{T. KWAN}

Y. FuJITA

Hokkaido University,

Sapporo.

Sept. 29.

${ }^{1}$ Kwan, T., J. Chem. Phys., 18, 1309 (1950). Kwan, T., and Fujita, Y., Bull. Chem. Soc. Japan, 24, 46 (1951).

${ }^{2}$ Brunauer, S., Fmmett, P. H., and Teller, E., J. Amer. Chem. Soc. 60, 309 (1938)

${ }^{8}$ Kwan, T., J. Res. Inst. Catalysie, Hokk. Univ., 1, No. 2, 81 (1949).

\section{Preparation of Single Crystals of Titanium and their Mode of Deformation}

SiNGLE crystals up to $20 \mathrm{~mm} . \times 2.5 \mathrm{~mm} . \times 1 \mathrm{~mm}$. have been prepared in pure titanium by a modified strain anneal technique. Pure titanium of hardness of $90 \mathrm{~V} . P . N$., indicating $0.01 \mathrm{wt}$. per cent of oxygen plus nitrogen ${ }^{1}$, prepared in this laboratory by the van Arkel process, was first arc-melted ${ }^{2}$ and- then cold-rolled with intermediate annealing at $860^{\circ} \mathrm{C}$. to strip in which there was very little preferred texture. By suitably gettering the purified argon used as a protective atmosphere throughout this preparation, the hardness of the titanium was not increased. The grain-size of this strip was fairly coarse and uneven (1-25 grains $/ \mathrm{mm}^{2}{ }^{2}$. Tensile strains from 0.05 to 5 per cent did not give large crystals on annealing for $90 \mathrm{hr}$. at $860^{\circ} \mathrm{C}$.; but compressive strains between 0.1 and 1 per cent, followed by similar annealing, produced in one-third of the specimens a crystal occupying the whole cross-section and an appreciable part of the gauge length, as illustrated.

In a less pure titanium (V.P.N. 165, 0.1 wt. per cent oxygen plus nitrogen ${ }^{1}$ ) the strip had an even grain-size of $20-25$ grains $/ \mathrm{mm} .{ }^{2}$. Compressive strains produced no appreciable grain growth on the annealing ; but a tensile strain of $1 \frac{1}{2}-2 \frac{1}{2}$ per cent resulted in single crystals.

It is suggested that the purity of the titanium influences the techniques required to produce single crystals because it affects the initial grain size of the strip. It is known that initial grain size is a critical factor in the technique of strain anneal growth".

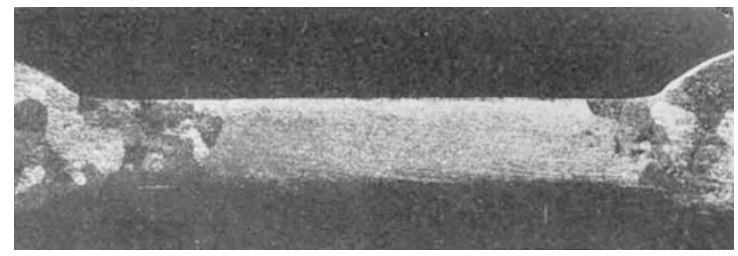

Single crystal of titanium, etched in 8 per cent nitric acid, 8 per cent hydrofluoric acid in water. $\times$ about 4

The size required in titanium (25 grains $/ \mathrm{mm}^{2}{ }^{2}$ ) is coarse in comparison with the size required in Armco iron ( 120 grains $/ \mathrm{mm}^{2}$ ) and is thought to be necessary to reduce deformation twinning during the critical straining. In a specimen with an initial grain size of 100 grains $/ \mathrm{mm}^{2}$ the critical straining induced a considerable amount of deformation twinning, and subsequent annealing produced no enhanced grain growth, but the deformation twins did disappear.

During tensile tests in a rigid machine at room temperature it has been shown that, when suitably oriented, single crystals of pure titanium slip on $(0001)[1 \overline{1} \overline{2} 0]$ as well as on $(10 \overline{1} 0)[11 \overline{2} 0]$ and $(10 \overline{1} 1)$ [11 $\overline{2} 0]$. Rosi, Dube and Alexander ${ }^{4}$ only observed slip on $(1 \overline{010})[1 \overline{2} 0]$ and $(10 \overline{1} 1)[11 \overline{2} 0]$ in work with coarse polycrystalline titanium made by the Kroll process. Slip on (0001) [11 $\overrightarrow{2} 0]$ has been shown to be required if the rolling textures in titanium are to be completely accounted for ${ }^{5}$. The critical resolved shear stress for slip on the basal plane appears to be higher

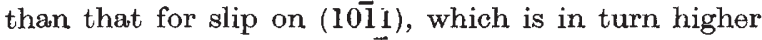
than that for slip on (1010). Deformation twinning on (10/2) and (11 $\overline{2} 1)$ has been observed. Deformation or kink bands are formed both during basal and (10) 10$)$ slip, and the bend plane has been identified as the $(11 \overline{2} 0)$ plane at right angles to the operative slip direction. The bent region of the lattice at the boundary of the band exhibits asterism, while the slipped region outside and inside the band does not. On annealing, the bent region polyganizes as shown by the break up of the asterism into discrete spots and the appearance of boundaries at right angles to the slip lines on the micro structure.

The stress-strain curve at room temperature shows a drop in load required to maintain plastic flow reminiscent of the yield phenomena in iron, but which appears to coincide with the appearance of the deformation band.

A more complete survey of these results is in the course of preparation for publication.

I wish to thank Dr. G. A. Geach, leader of the Physical Metallurgy Section of this laboratory, for encouragement and helpful discussion; I also wish to express my thanks to Dr. T. E. Allibone for permission to publish this paper.

Associated Electrical Industries, Ltd.

\section{A. T. Churchman}

Research Laboratory,

Aldermaston Court,

Aldormaston, Berkshire. April 9.

${ }^{1}$ Cross, U. C., O.N.R. Symposium on Titanium, Washington, 1949 Jenkins and Worner, J. Inst. Met., 80, 157 (1951).

${ }^{2}$ Geach, G. A., and Summers-Smith, J. D., Metallurgia, 42, 153 (1950). 'Edwards, C. A., and Pfeil, L. B., J. Iron and Steel Inst. (II), 109, $129(1924)$.

- Rosi, F. D., Dube, C. A., and Alexander, B. H., J. Metals, 5, 257 (1953).

${ }^{8}$ Williams, D. N., and Eppelsheimer, D. S., Nature, 170, 146 (1952). 